\title{
Correspondence
}

\section{An Efficient Method for Quantifying the Multichannel EEG Spatial-Temporal Complexity}

Pei-Chen Lo* and Wen-Po Chung

\begin{abstract}
The complexity index $(\delta)$ quantifies the intrinsic dimensionality of the global complexity of a point set, and was shown to be able to characterize electroencephalogram spatial-temporal features. The complexity index is conceptually comprehensible and easily implemented, yet, it is time consuming. In this paper, we present an efficient computational method based on the projection of the high-dimensional state-space points onto a one-dimensional axis. The computational time decreases by at least $50 \%$, without affecting the measure accuracy.
\end{abstract}

Index Terms-Complexity index, global waveform complexity, intrinsic dimension, multichannel EEG (electroencephalogram), spatial-temporal feature, state-space trajectory.

\section{INTRODUCTION}

The electroencephalogram (EEG) is an important clinical tool for diagnosing and monitoring the nervous system regarding normal or pathological conditions. The temporal and spatial EEG features provide a basis for the detection of focal pathologies [1]. To deal with an enormous amount of recorded EEG, data quantification has been the primary step of EEG analysis. In addition to the popular time-domain and frequency-domain approaches, other techniques have been introduced and proved useful in EEG analysis [2]. Among them, methods based on the nonlinear dynamical theory have been used for over a decade to quantify the underlying brain dynamics and evaluate the EEG waveform complexity [3]-[6]. Nonetheless, tools from nonlinear dynamical theory used for EEG analysis, such as the dimensional computation and Lyapunov exponent estimation, mostly suffer from computational inefficiency and bias from implementing parameters [4], [7]. Thus, they are not yet feasible for long-term monitoring in small computers. On the other hand, the spatial EEG features over the scalp surface are of great importance. Destexhe et al. [8] and Dvorak [9] proposed to estimate the correlation dimension of multichannel EEG by using the number of recording sites as embedding dimension of the state space [10]. Then the $n$-channel EEG data were viewed as a trajectory in $n$-dimensional state space. In this way they were able to evaluate the "global" correlation dimension quantifying the spatial-temporal feature of EEG. Since evaluating the correlation dimension involves slope computation using linear regression, an appropriate linear scaling region needs to be determined first. This kind of indirect estimation procedure is a crucial drawback in practical situations.

The topological or intrinsic dimensionality of a point set was introduced to characterize data sets in the field of pattern recognition [11]-[14]. To improve the computational efficiency, methods for determining the intrinsic dimension were mostly based on local

Manuscript received August 19, 1999; revised October 19, 2000. This work was supported by the National Science Council of Taiwan under Grant, NSC892213-E-009-186. Asterisk indicates corresponding author.

*P.-C. Lo is with the Department of Electrical and Control Engineering, National Chiao Tung University, 1001 Ta-Hsueh Road, Hsinchu 30010, Taiwan, R.O.C. (e-mail: pclo@cc.nctu.edu.tw).

W.-P. Chung is with the Department of Electrical and Control Engineering, National Chiao Tung University, Taiwan, R.O.C.

Publisher Item Identifier S 0018-9294(01)01580-4. approaches, that is, quantifying the local features in small regions [13], [15]-[17]. Among them, the $K$ 's nearest neighborhood ( $K \mathrm{NN}$ ) analysis provides an approach for directly estimating intrinsic dimensionality [12], [15], [18]. The method is conceptually simple, yet, has a drawback of spending an enormous amount of time on exhaustive searches for the $K N N$ distance. Thus, we develop a new algorithm for searching the $K \mathrm{NN}$ distance, which saves a lot of computer time. When adapted to multichannel EEG analysis to explore the spatial-temporal characteristics, the intrinsic dimensionality reflects variations in the degree of waveform complexity in a data set. Hence the estimated result is to be called "complexity index $(\delta)$."

\section{AN IMPROVED $K$ NN ALGORITHM FOR EVALUATING $\delta$}

Let $\mathbf{X}=\left\{\mathbf{X}_{i}\right\}_{i=1}^{N}$ be the set of points on the EEG trajectory, where $\mathbf{X}_{i}$ is an $n$-dimensional point constructed from the $n$-channel EEG signals. For instance, $\mathbf{X}_{i}=(\mathrm{F} 3(i), \mathrm{F} 4(i), \mathrm{Cz}(i), \mathrm{P} 3(i), \mathrm{P} 4(i))$ represents a point on the five-dimensional space, whose coordinates (degrees of freedom) are brain electrical potentials recorded from sites F3, F4, Cz, P3 and P4, respectively. For each point in the set $\mathbf{X}$ (e.g., $\mathbf{X}_{i}$ ), a $K$ NN hypersphere is determined and formed by the $K$ 's nearest neighboring (NN) points $\left\{\mathbf{V}_{i j}\right\}_{j=1}^{K}, \mathbf{V}_{i j} \in \mathbf{X}$, and $\mathbf{V}_{i 1}=\mathbf{X}_{i}$. The $\mathbf{X}_{i}$ is called the seed point of the $i$ th hypersphere. Inside the $i$ th hypersphere, the largest distance to the seed point $\mathbf{X}_{i}$ is

$$
d_{i, K \mathrm{NN}}=\left\|\mathbf{V}_{i K}-\mathbf{X}_{i}\right\|
$$

where the operator $\|\cdot\|$ evaluates the Euclidean distance. Fukunaga and Flick [15] analyzed the pattern classification error and obtained

$$
\frac{E\left\{d_{(K+1) \mathrm{NN}}\right\}}{E\left\{d_{K \mathrm{NN}}\right\}}=1+\frac{1}{K n}
$$

where $E\left\{d_{K \mathrm{NN}}\right\}$ is the first-order moment of $d_{K \mathrm{NN}}$. The $d_{K \mathrm{NN}}$ denotes the $K$ th NN distance of any hypersphere in $\mathbf{X}$. Equation (2) points out the effect of $K$ on classification error for a given number of space dimension $n$. Fukunaga and Flick aimed at quantifying the pattern classification error in the $K N \mathrm{NN}$ model. They hypothesized an $n$-dimensional space for the pattern vectors, where $n$ is an integer. In order to quantify the global waveform complexity of multichannel EEG, we apply the concept of fractional dimensionality of a strange attractor. Following their work, we derived the equation for evaluating the complexity index $\delta$ as follows [19]:

$$
\delta=\frac{1}{K}\left(\frac{E\left\{d_{(K+1) \mathrm{NN}}\right\}}{E\left\{d_{K \mathrm{NN}}\right\}}-1\right)^{-1} .
$$

Equation (3) is actually implemented in the algorithm as

$$
\delta=\frac{1}{K}\left(\frac{\overline{d_{(K+1) \mathrm{NN}}}}{\overline{d_{K \mathrm{NN}}}}-1\right)^{-1}
$$

where $\overline{d_{(K+1) \mathrm{NN}}}$ and $\overline{d_{K \mathrm{NN}}}$ are the temporal average of $d_{i,(K+1) \mathrm{NN}}$ and $d_{i, K \mathrm{NN}}$, respectively, for $i=1, \ldots, N$. Apparently, $\delta$ may be a fractional number.

One advantage of the complexity index is the easy implementation of computing (4). A large portion of the computer time is spent on searching for the $K \mathrm{NN}$ and $(K+1) \mathrm{NN}$ distances. To find $d_{i, K N N}$ and $d_{i,(K+1) \mathrm{NN}}$ for the seed point $\mathbf{X}_{i}$, an intuitive 
approach is to create and then dynamically update a data buffer array $\boldsymbol{\Delta}=\left\{\Delta_{i, 1}, \ldots, \Delta_{i, K}, \Delta_{i, K+1}\right\}$ so that $\boldsymbol{\Delta}$ always stores the $(K+1)$ 's smallest distances in sequence when a new interpoint distance is computed. The detailed strategy is explained as follows. Consider that the seed point is $\mathbf{X}_{i}$. The algorithm in the beginning sorts the first $(K+1)$ 's interpoint distances, $d_{i j}=\left\|\mathbf{X}_{j}-\mathbf{X}_{i}\right\|$, $j=1, \ldots,(K+1)$, and keeps them in sequence in the data buffer $\boldsymbol{\Delta}$. The elements in the sorted data array satisfy: $\Delta_{i, m} \leq \Delta_{i, n}$ if $1 \leq m<n \leq(K+1)$. Each of the remaining distances, $d_{i j}, j=(K+2), \ldots, N$, is then compared with every element in $\boldsymbol{\Delta}$. If $\Delta_{i, m-1}<d_{i j}<\Delta_{i, m}, d_{i j}$ is inserted in the $m$ th position and the $\Delta_{i, K+1}$ is excluded from the buffer. The competition process is performed on each newly computed interpoint distance. Finally, the data buffer stores the smallest $(K+1)$ 's distances. Accordingly, $d_{i, K \mathrm{NN}}=\Delta_{i, K}$ and $d_{i,(K+1) \mathrm{NN}}=\Delta_{i, K+1}$. The complexity index $\delta$ is then computed by using (4). Undoubtedly, computer time required by the algorithm implemented in this manner is highly dependent on the values of $K$ and $N$. A large $K$ costs more effort in the competition process. A large $N$ indicates a large number of distances to be computed.

In this paper, the authors propose an approach that does not require computing all the interpoint distances and reduces the exhaustive sorting process. The computer time can be significantly reduced especially for a large number of data points $(N)$. Let $[\mathbf{X}]$ be an $N \times n$ matrix with its $i$ th row vector representing the $i$ th $n$-dimensional point $\mathbf{X}_{i}$ on the EEG trajectory. The eigenvector associated with the largest eigenvalue of the covariance matrix of $[\mathbf{X}]$ is denoted by $[\Phi]$, a $1 \times n$ row matrix. And $\|\mathbf{\Phi}\|=1$. Then the transformation $[\mathbf{Y}]=[\mathbf{X}][\boldsymbol{\Phi}]^{\mathrm{T}}$ results in an $N \times 1$ column matrix containing $N$ scalars $y_{i}, i=1, \ldots, N$. In other words, the transformation projects the $n$-dimensional state-space points onto the principal axis derived from the largest eigenvector. As a result, the interpoint distances of $\mathbf{X}_{i}: d_{i j}, j=1, \ldots, N$ are mapped to

$$
\rho_{i j}=\left\|y_{j}-y_{i}\right\|=\left\|\left(\mathbf{X}_{j}-\mathbf{X}_{i}\right) \Phi^{\mathrm{T}}\right\|
$$

which implies

$$
\rho_{i j}=\left\|y_{j}-y_{i}\right\| \leq\left\|\mathbf{X}_{j}-\mathbf{X}_{i}\right\|=d_{i j}
$$

according to the Cauchy-Schwartz inequality. Using $\rho_{i j}$ as reference, the sorting process for determining the $d_{i, K \mathrm{NN}}$ and $d_{i,(K+1) \mathrm{NN}}$ becomes less laborious. Let $[\mathbf{S}]$ denote the sorted column matrix of $[\mathbf{Y}]$ so that its elements satisfy $s_{1} \leq s_{2} \leq \cdots \leq s_{N}$. Fig. 1 illustrates the transformation process. We shall describe the proposed method for finding the $d_{i, K N N}$ and $d_{i,(K+1) \mathrm{NN}}$ by using Fig. 2 . Assume that $s_{p}=y_{i}$, that is, $y_{i}$ is in the $p$ th order on the principal axis following the transforming process

$$
\mathbf{X}_{i} \stackrel{\Phi}{\longrightarrow} y_{i} \stackrel{\text { sorting }}{\longrightarrow} s_{p}
$$

Then the algorithm examines those points in the neighborhood of $s_{p}$ : $\left\{s_{p \pm 1}, s_{p \pm 2}, \ldots\right\}$. For each neighboring point, its associated $n$-dimensional point in $[\mathbf{X}]$ and the state-space distance from $\mathbf{X}_{i}$ can be determined. The computed distances are stored in sequence in a data buffer. Assume that, after examining $M$ neighboring points within the dotted frame $(M \geq K+1)$, the largest $(K+1)$ NN distance is obtained, which is $d_{i,(K+1) \mathrm{NN}}=\left\|\mathbf{X}_{k}-\mathbf{X}_{i}\right\|$. The algorithm continues examining, in two-sided direction, the points on the principal axis until $\left\|s_{r}-s_{p}\right\| \geq d_{i,(K+1) \mathrm{NN}}$ (left) and $\left\|s_{t}-s_{p}\right\| \geq d_{i,(K+1) \mathrm{NN}}$ (right). This condition ensures that the remaining points are all farther than $d_{i,(K+1) \mathrm{NN}}$ since

$$
d_{i,(K+1) \mathrm{NN}} \leq\left\|s_{r}-s_{p}\right\|=\left\|y_{l}-y_{i}\right\| \leq\left\|\mathbf{X}_{l}-\mathbf{X}_{i}\right\|
$$

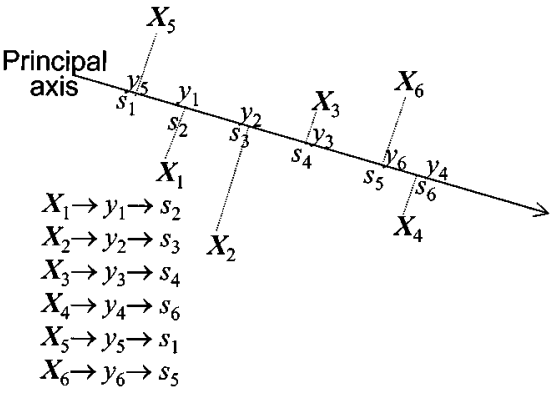

Fig. 1. The transformation process that maps six $n$-dimensional points onto the principal axis and re-indexes the one-dimensional (1-D) array.

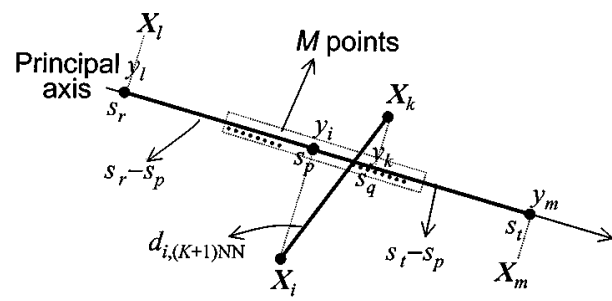

Fig. 2. The search for $d_{i, K N \mathrm{~N}}$ and $d_{i,(K+1) \mathrm{NN}}$ is completed when the left and right boundary points $s_{r}$ and $s_{t}$, satisfying (8), are found.

and

$$
d_{i,(K+1) \mathrm{NN}} \leq\left\|s_{t}-s_{p}\right\|=\left\|y_{m}-y_{i}\right\| \leq\left\|\mathbf{X}_{m}-\mathbf{X}_{i}\right\|
$$

according to (6). The search for $d_{i, K N N}$ and $d_{i,(K+1) \mathrm{NN}}$ is completed at this point. As illustrated in Fig. 2, two points $s_{r}$ and $s_{t}$ define the left and right boundary of the searching region on the principal axis. Note that, given a seed point $s_{p}$, the number of points being searched before satisfying (8) cannot be estimated by some a priori knowledge of the EEG space trajectory. Thus, computational complexity cannot be explicitly determined by parameters like $N, K$, and $n$. An empirical approach for evaluating the computational efficiency is applied (Section III).

To validate the accuracy of the developed method, Table I lists the average $\delta$ (denoted by $\bar{\delta}$ ) evaluated for the model-generated trajectory. The correlation dimension reported in [20] is shown for comparison. The $\bar{\delta}$ was computed by averaging the $\delta$ s over a moderate range of values of $K(30 \leq K \leq 60)$ to obtain a reliable estimate. The resulting $\bar{\delta}$ approximates well the correlation dimension except for the Zaslavskii map. Next, we apply the proposed method to the multichannel EEG signals and compare its computational efficiency with that of the straightforward algorithm [see, (4)]. Both algorithms were programmed in Turbo $C$. The following results were obtained by executing the algorithms on a Pentium-133 notebook computer.

\section{RESUltS OF EFFICIENCY COMPARISON}

Our previous work has demonstrated the capability of $\bar{\delta}$ in characterizing the spatial-temporal feature of the multichannel EEG signals [19]. In the paper, the EEG data analyzed were recorded from a patient who had an epilepsy syndrome called "Benign Partial Epilepsy of Childhood," yet had never had obvious epileptic seizures (provided by Dr. F. Matsuo, Department of Neurology, University of Utah Medical School). Prominent focal-sharp-wave transients appeared several times. The patient was sleeping during the recording. A 25-channel electrode array, including 19 channels in the 10-20 system and six south-hemispherical channels [21], with a common linked-ear reference was applied. The sampling rate was $200 \mathrm{~Hz}$. In [19], we showed that the running $\bar{\delta}$ curve (window length: 1000 samples, moving size: 
TABLE I

$\bar{\delta}$ Estimated FOR MODEl-GENERATED TRAJECTORIES

\begin{tabular}{l|c|c|c||c|c}
\hline & $\bar{\delta}$ & $N / \Delta t$ & range of $K$ & $\begin{array}{l}\text { Correlation } \\
\text { Dimension }\end{array}$ & $N / \Delta t$ \\
\hline $\begin{array}{l}\text { Rabinovich- } \\
\text { Fabrikant model }\end{array}$ & 2.19 & $15,000 / 0.25$ & $30 \sim 60$ & 2.18 & $15,000 / 0.25$ \\
\hline Lorenz model & 2.04 & $15,000 / 0.25$ & $30 \sim 60$ & 2.05 & $15,000 / 0.25$ \\
\hline Zaslavskii map & 1.79 & 25,000 & $30 \sim 60$ & $\sim 1.50$ & 25,000 \\
\hline Hénon map & 1.25 & 15,000 & $30 \sim 60$ & 1.25 & 15,000 \\
\hline
\end{tabular}

$\Delta t$ a fixed time increment.

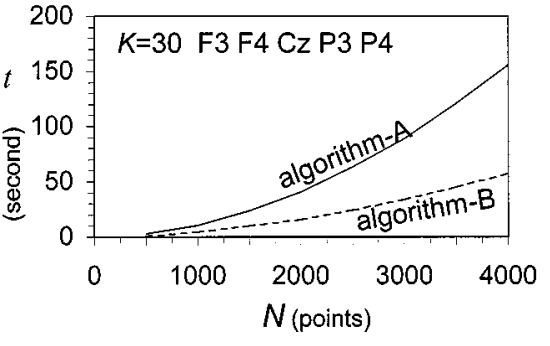

Fig. 3. Efficiency comparison for a five-channel EEG with variable lengths $(K=30)$.

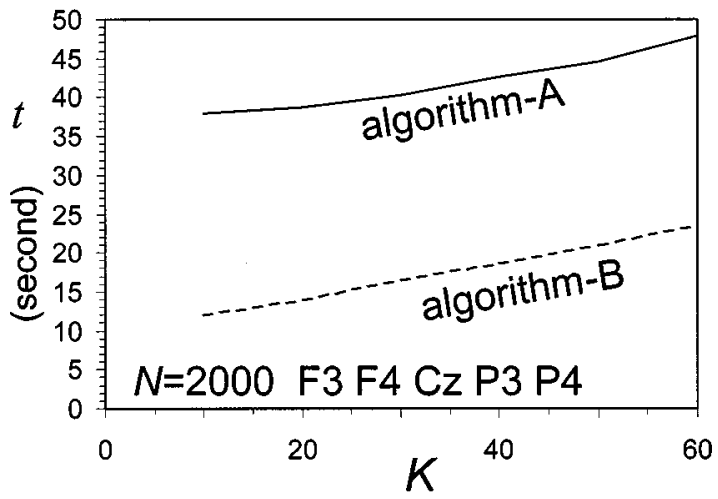

Fig. 4. Efficiency comparison for a five-channel EEG using variable $K \mathrm{~s}(N=$ 2000).

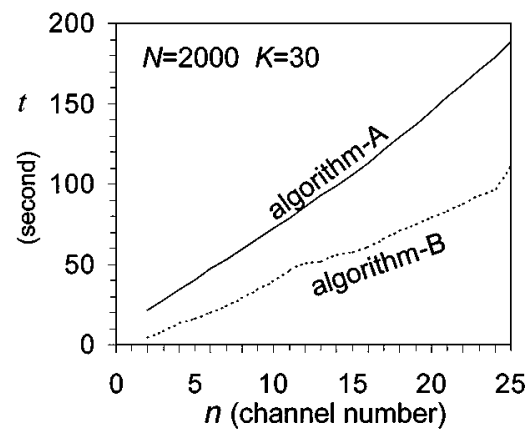

Fig. 5. Efficiency comparison for variable channel numbers $(K=30$ and $N=2000)$.

100 samples, $25 \leq K \leq 35$ ) was able to identify occurrence of EEG focal-sharp-wave events and quantify EEG spatial correlation. The background EEG had a complexity index between 4.0 and 4.5,

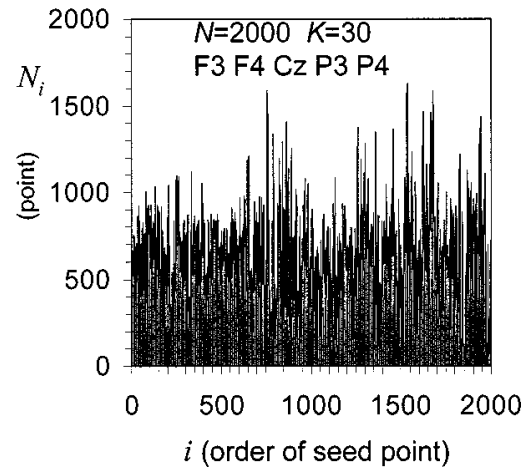

Fig. 6. Number of interpoint distances required to obtain $d_{i, K \mathrm{NN}}$ and $d_{i,(K+1) \text { Nv }}$ for each seed point.

which was reduced to $3.0 \sim 3.5$ when focal-sharp-wave transient occurred. The analysis demonstrated that the running $\bar{\delta}$ curve might be used to detect the occurrence of particular events in the EEG monitoring. On the other hand, an array composed of channels with quite different waveform patterns (spatially uncorrelated) tended to have a larger $\bar{\delta}$. Thus, the $\bar{\delta}$ analysis may be a tool to study the spatial correlation in the multichannel EEG signals.

In this paper, we aim to validate the efficiency of the proposed algorithm. The two algorithms presented in Section II will be referred to as algorithm-A (the straightforward implementation) and algorithm-B (the efficient method). The EEG signal is the same one that was analyzed in [19]. First, a five-channel protocol, involving electrode sites $\mathrm{F} 3, \mathrm{~F} 4, \mathrm{Cz}, \mathrm{P} 3$, and $\mathrm{P} 4$, is used to compare the efficiency of two algorithms for different values of $N, K$, and $n$ (Figs. 3-5). For increasing $N$, this reduction becomes more evident. The efficient algorithm (B) reduces the computational time to less than half the time required by algorithm-A at 1500 samples, and it has a much slower slope. The algorithm-A undoubtedly needs to evaluate $N \times(N-1)$ interpoint distances for an $N$-point state-space EEG trajectory. The algorithm-B spends less than $10 \%$ of the total computational time to find the principal axis and to project the $n$-dimensional state-space points onto the principal axis. Most of the computational time is consumed by the computation of interpoint distances. However, unlike the algorithm-A, the number of interpoint distances being computed by the algorithm-B cannot be equated. An empirical approach is applied to show how the algorithm-B achieves its computational efficiency. Let $N_{i}$ denote the number of interpoint distances being computed by algorithm-B in order to obtain $d_{i, K N N}$ and $d_{i,(K+1) \mathrm{NN}}$ for each seed point $\mathbf{X}_{i}, i=1, \ldots, N,(N=2000, K=30)$. Note that $N_{i}=N-1$ for all $i$ if applying algorithm-A. The result for algorithm-B is plotted in Fig. 6. As addressed in Section III, there exists no explicit relation 
between the number of points being searched and the parameters of the EEG trajectory, which is corroborated in this figure. As shown in Fig. 6, most $N_{i}$ are smaller than $1000(N / 2)$. The average of $N_{i}, \bar{N}_{i}$, is 740 . Consequently, the overall computational time can be reduced by at least one half. Finally, according to our experience and the manner $K \mathrm{NN}$ distance is computed, the computational time required is approximately the same for any given $K, N$, and $n$, independent of the EEG spatial-temporal complexity.

\section{CONCLUSION}

This paper demonstrated an efficient methodology for computing the intrinsic dimensionality used to quantify the spatial-temporal feature of the multichannel EEG signals. The $n$-dimensional points on the EEG trajectory (the set $[\mathbf{X}]$ ) were first projected onto the principal axis derived from the largest eigenvector of the covariance matrix of $[\mathbf{X}]$. The order and the distances of the projected 1-D points provide a guideline for the algorithm to confine its searching scope to a smaller region. The algorithm need not perform the exhausting search for all $K \mathrm{NN}$ distances. The computational time, thus, decreases substantially.

\section{REFERENCES}

[1] T. Kalayci and Ö Özdamar, "Wavelet preprocessing for automated neural network detection of EEG spikes," IEEE Eng. Med. Biol. Mag., vol. 14, no. 2, pp. 160-166, 1995

[2] M. v. Gils, A. Rosenfalck, S. White, P. Prior, J. Gade, L. Senhadji, C. Thomsen, I. R. Ghosh, R. M. Langford, and K. Jensen, "Signal processing in prolonged EEG recordings during intensive care," IEEE Eng. Med. Biol. Mag., vol. 16, no. 6, pp. 56-63, 1997.

[3] A. Babloyantz and A. Destexhe, "Low-dimensional chaos in an instance of epilepsy," Proc. Nat. Acad. Sci. USA, vol. 83, pp. 3513-3517, 1986.

[4] P.-C. Lo and J. C. Principe, "Dimensionality analysis of EEG segments: Experimental considerations," in Proc. Int. Joint Conf. Neural Network Soc., 1989, pp. 693-698.

[5] P. E. Rapp, T. R. Bashore, J. M. Martinerie, A. M. Albano, and A. I. Mees, "Dynamics of brain electrical activity," Brain Topogr., vol. 2, pp. 99-118, 1989.

[6] J. P. M. Pijn, J. V. Neerven, A. Noest, and F. H. Lopes da Silva, "Chaos or noise in EEG signals: Dependence on state and brain site," Electroenceph. Clin. Neurophysiol., vol. 79, pp. 371-381, 1991.

[7] I. Yaylali, H. Koçak, and P. Jayakar, "Detection of seizures from small samples using nonlinear dynamic system theory," IEEE Trans. Biomed. Eng., vol. 43, pp. 743-751, July 1996.

[8] A. Destexhe, J. A. Sepulchre, and A. Babloyantz, "A comparative study of the experimental quantification of deterministic chaos," Phys. Lett. A, vol. 132, pp. 101-106, 1988.

[9] I. Dvorak, "Takens versus multichannel reconstruction in EEG correlation exponent estimations," Phys. Lett. A, vol. 151, pp. 225-233, 1990.

[10] J. Wackermann, D. Lehmann, I. Dvorak, and C. M. Michel, "Global dimensional complexity of multi-channel EEG indicates change of human brain functional state after a single dose of a nootropic drug," Electroenceph. Clin. Neurophysiol., vol. 86, pp. 193-198, 1993.

[11] K. Fukunaga and D. R. Olsen, "An algorithm for finding intrinsic dimensionality of data," IEEE Trans. Comput., vol. C-20, pp. 176-183, Feb. 1971.

[12] K. W. Pettis, T. A. Bailey, A. K. Jain, and R. C. Dubes, "An intrinsic dimensionality estimator from near-neighbor information," IEEE Trans. Pattern Anal. Machine Intell., vol. PAMI-1, pp. 25-37, Jan. 1979.

[13] P. J. Verveer and P. W. Duin, "An evaluation of intrinsic dimensionality estimators," IEEE Trans. Pattern Anal. Machine Intell., vol. 17, pp. 81-86, Jan. 1995.

[14] J. Bruske and G. Sommer, "Intrinsic dimensionality estimation with optimally topology preserving maps," IEEE Trans. Patt. Anal. Machine Intell., vol. 20, no. 5, pp. 572-575, 1998.

[15] K. Fukunaga and T. E. Flick, "Classification error for a very large number of classes," IEEE Trans. Pattern Anal. Machine Intell., vol. PAMI-6, pp. 779-788, June 1984.

[16] A. Passamante and M. E. Farrell, "Characterizing attractors using local intrinsic dimension via higher-order statistics," Phys. Rev. A, vol. 43, no. 10 , pp. 5268-5274, 1991
[17] O. Michel and P. Flandrin, "Local minimum redundancy representation of a system for estimating the number of its degrees of freedom," in Proc. IEEE Signal Processing Workshop Higher-Order Statistics, 1993, pp. 341-345.

[18] G. V. Trunk, "Statistical estimation of the intrinsic dimensionality of a noisy signal collection," IEEE Trans. Comput., vol. C-25, pp. 165-171, Feb. 1976.

[19] P.-C. Lo and W.-P. Chung, "An approach to quantifying the multi-channel EEG spatial-temporal feature," Biomaterial J., vol. 42, no. 7, pp. 901-916, May 2000.

[20] P. Grassberger and I. Procaccia, "Characterization of strange attractors," Phys. Rev. Lett., vol. 50, no. 5, pp. 346-349, 1983.

[21] F. Matsuo, "Expanded head surface EEG electrode array: An application to display the voltage topography of focal epileptiform discharges of mesiotemporal origin," J. Clin. Neurophysiol., vol. 8, no. 4, pp. 442-451, 1991.

\section{A Self-Oscillating Detuning-Insensitive Class-E Transmitter for Implantable Microsystems}

\author{
Babak Ziaie*, Steven C. Rose, Mark D. Nardin, and Khalil Najafi
}

Abstract-This paper describes a low-cost, self-oscillating, detuning-insensitive, class-E driver for transcutaneous power and data transmission to implantable microsystems. A voltage feedback scheme using a fast comparator for zero-crossing detection and a CMOS start-up circuit were used to stabilize the class-E operation for various transmitter coil inductance values. This technique solves the common problem of mismatch between the switching frequency of the driving device and the resonant frequency of the load network, which can cause excessive power loss and damage to the active device. Data is transmitted by AM modulation of the carrier through switching the power supply between two levels. The transmitter uses a 9-V supply, consumes $212 \mathrm{~mA}$, operates at 3.9 $\mathrm{MHz}$, and has an efficiency of $71 \%$. The efficiency is stable $(<2 \%$ change $)$ against $13 \%$ variations in the inductance value of a pancake shaped transmitter coil.

Index Terms-Biomedical microsystems, class-E transmitter, implantable electronics, inductive powering, transcutaneous links.

\section{INTRODUCTION}

Telemetry inductive powering is an attractive alternative to batteries in implantable devices that require extended operation lifetime [1]-[3]. The telemetry link in most of these applications is a transformer-like coupled pair of coils that has been previously optimized for power transfer efficiency [4]. Recent advances in micromachining and microtechnology have enabled investigators to fabricate miniature implantable devices [5], [6]. Due to the small size of the receiver coil (1-mm diameter, 3-mm length in [6]), the power transmission efficiency in many of these microdevices is rather low [with the implanted system receiving $<1 \%$ of the average emitted radio-frequency (RF) energy]. In order to supply enough power to the receiver coil, a high-efficiency transmitter/amplifier must be used. Class-E power amplifiers

Manuscript received September 9, 1999; revised November 16, 2000. This work was supported by the Neural Prosthesis Program, National Institutes of Health (NIH), under Contracts NIH-N01-NS-4-2319, NIH-N01-NS-1-2314, and NIH-N01-NS-8-2312. Asterisk indicates corresponding author.

*B. Ziaie is with the Department of Electrical and Computer Engineering, University of Minnesota, 200 Union Street SE, Minneapolis, MN 55455 USA.

S. C. Rose and K. Najafi are with the Department of Electrical Engineering and Computer Science, University of Michigan, Ann Arbor, MI 48109 USA.

M. D. Nardin is with the Intel Corporations, Hillsboro, OR 97124 USA.

Publisher Item Identifier S 0018-9294(01)01581-6. 